\title{
Asthma and affective traits in adults: a genetically informative study
}

\author{
Kelli Lehto ${ }^{1,2}$, Nancy L. Pedersen ${ }^{1}$, Catarina Almqvist ${ }^{1,3}$, Yi Lu $^{1}$ and \\ Bronwyn K. Brew ${ }^{1}$
}

Affiliations:

${ }^{1}$ Dept of Medical Epidemiology and Biostatistics, Karolinska Institutet, Stockholm, Sweden.

${ }^{2}$ Dept of Chronic Diseases, Institute for Health Development, Tallinn, Estonia.

${ }^{3}$ Astrid Lindgren Children's Hospital, Karolinska University Hospital, Stockholm, Sweden.

Correspondence:

Kelli Lehto, Dept of Medical Epidemiology and Biostatistics, Karolinska Instituet, Nobels Vag 12a, 17177. Sweden.

E-mail: kelli.lehto@ki.se

@ERSpublications

This study found evidence for shared genetic liability between asthma, depression and neuroticism. Physicians should be aware that asthma patients might also suffer from depression and emotional instability because of common underlying pathways. http://ow.ly/wnoC30o7vZT

Cite this article as: Lehto K, Pedersen NL, Almqvist C, et al. Asthma and affective traits in adults: a genetically informative study. Eur Respir J 2019; 53: 1802142 [https://doi.org/10.1183/13993003.02142-2018].

ABSTRACT Depression, anxiety and high neuroticism (affective traits) are often comorbid with asthma. A causal direction between the affective traits and asthma is difficult to determine; however, there may be a common underlying pathway attributable to shared genetic factors. Our aim was to determine whether a common genetic susceptibility exists for asthma and each of the affective traits.

An adult cohort from the Swedish Twin Registry underwent questionnaire-based health assessments $(\mathrm{n}=23693)$ and genotyping $(\mathrm{n}=15908)$. Firstly, questionnaire-based associations between asthma and affective traits were explored. This was followed by genetic analyses: 1) polygenic risk scores (PRS) for affective traits were used as predictors of asthma in the cohort, and 2) genome-wide association results from UK Biobank were used in linkage-disequilibrium score regression (LDSC) to quantify genetic correlations between asthma and affective traits. Analyses found associations between questionnaire-based asthma and affective traits (OR 1.67, 95\% CI 1.50-1.86 major depression; OR 1.45, 95\% CI 1.30-1.61 anxiety; and OR 1.60, 95\% CI 1.40-1.82 high neuroticism). Genetic susceptibility for neuroticism explained the variance in asthma with a dose-response effect; that is, study participants in the highest neuroticism PRS quartile were more likely to have asthma than those in the lowest quartile (OR 1.37, 95\% CI 1.17-1.61). Genetic correlations were found between depression and asthma ( $\mathrm{rg}=0.17)$, but not for anxiety or neuroticism.

We conclude that the observed comorbidity between asthma and the affective traits may in part be due to shared genetic influences between asthma and depression (LDSC) and neuroticism (PRS), but not anxiety. 


\section{Introduction}

Asthma is a complex disease involving inflammation of the lungs and airways. It is the most common chronic respiratory disease in adults worldwide with a prevalence of $4-8 \%[1,2]$. Adults with asthma have twice as high risk of also having depression or anxiety compared with the general population [3-7]. This can lead to lowered quality of life and worse health outcomes. In particular, having depression can lead to poor adherence to inhaled corticosteroids, poor asthma control and increased asthma exacerbations [8-10]. Despite a number of studies investigating the link between asthma, depression and anxiety, direction of causality has been difficult to determine [11], hence leading to the conclusion that perhaps a common underlying pathway exists [12].

Similarly, several studies have shown that the personality trait neuroticism influences the risk for asthma in adults, possibly increasing the risk up to three times among those with high neuroticism scores [13-15]. Neuroticism is characterised by a tendency to experience emotional instability and excessive worry, and has notable stability across the lifespan $[16,17]$. It is also often a predecessor of depression and anxiety, which are considered to be psychiatric diagnoses that can change throughout a person's lifetime. All three affective traits show considerable correlations on both phenotypic and genotypic levels $[18,19]$.

A common genetic pathway seems to be a plausible explanation for co-occurrence between asthma and the affective traits especially as adult asthma is moderately heritable, as are depression, anxiety and neuroticism [20-22]. A Finnish study of adult twins found some evidence for a genetic association between asthma and anxiety/depression [23]. Our own familial aggregation study in twin children suggested a common underlying cause for asthma and anxiety/depression, however, a genetic hypothesis was not supported [24]. Both of these studies relied on twin modelling based on within-pair comparisons rather than using genotypic information. Furthermore, a shared genetic susceptibility between asthma and neuroticism has not been investigated at the molecular genetic level to date. More direct genetic research is needed to assess whether there is genetic variance shared between affective traits and asthma using recent information from powerful genome-wide association studies (GWASs) for each of these conditions.

The objectives of this study are to: 1) confirm associations between asthma and affective traits (depression, anxiety and neuroticism); and 2) investigate whether there is evidence for a shared genetic relationship between each of the affective traits and asthma in adults. This will be done by using results from large GWASs to investigate cross-trait genetic association using a polygenic risk score (PRS) approach and to estimate genetic correlations between traits using linkage disequilibrium score regression (LDSC).

\section{Methods}

\section{Study population}

The Swedish Twin Registry was chosen as the study population as it provided both phenotypic and genotypic information from adults on affective traits and asthma for a large number of individuals. Data were derived from three data collection occasions in the Swedish Twin Registry (figure 1) [25-27]. In 1972-1973 like-sexed twins born 1926-1958 were mailed a questionnaire collecting demographic, medical, pollution exposure, life-style and personality information, including information about neuroticism. About 36000 twins responded (aged 15-47 years) [25]. These were followed up in 1998-2002 (ages 41-74 years) with a computer-assisted telephone interview, including questions regarding common complex diseases such as asthma, depression and anxiety. There was a $74 \%$ response rate of those alive and living in Sweden, $n=23693$ [26]. In addition, at this time, opposite-sex pairs were recruited for the telephone interview, increasing the number to a total of 38633 participants. Between 2002 and 2010 (ages 44-82 years) these same twins that were still alive and had not participated in other genetic studies were asked to donate blood for bio-banking and genotyping purposes [27]. Approximately half of those contacted gave blood samples [27] Genotype information is now available for 15902 of these.

\section{Measures \\ Asthma}

Asthma is a derived variable based on questions reflecting current or previous asthma and allergy. We scored positive for asthma participants that either reported that their asthma had been diagnosed by a doctor or they had probable asthma. To be positive for probable asthma, the subject had to have one of: 1) self-reported asthma, whistle breathing, breathlessness at rest or breathlessness on waking up; and one of 2) asthma triggered by pollen, fur or hay, asthma onset between 4 and 30 years of age, self-reported hay fever with a debut between 4 and 30 years of age, asthma onset aged $<4$ years and still have asthma/age last had asthma was 4-20 years [28]. 


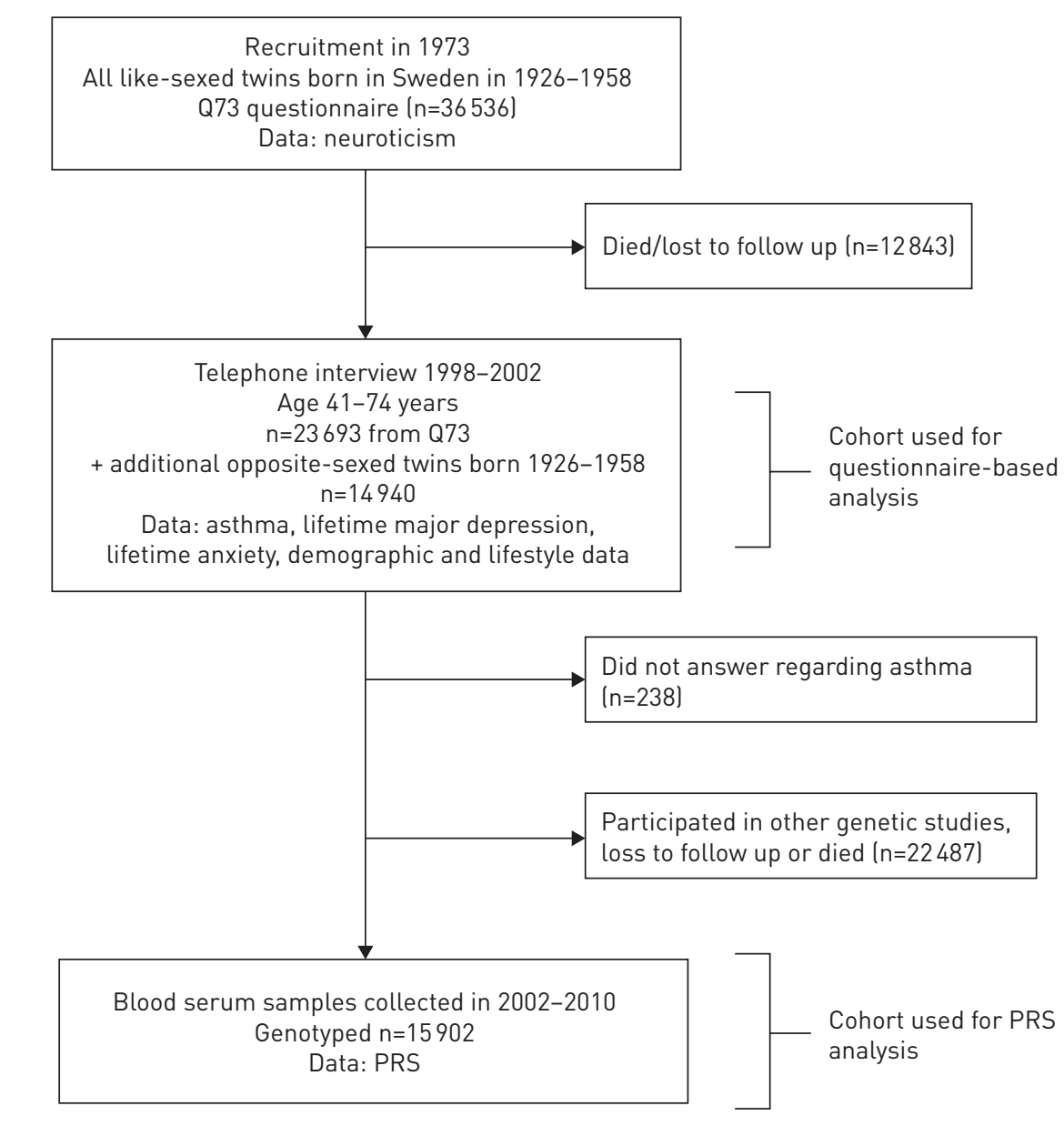

\begin{tabular}{|} 
UK Biobank GWAS summary results \\
GWAS range $\mathrm{n}=322668-337159$ \\
Phenotypes: neuroticism, self-report depression and \\
anxiety, seen a GP or psychiatrist for nerves, anxiety, \\
tension or depression
\end{tabular}

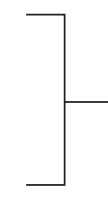

Cohort used for LDSC analysis

FIGURE 1 Flowchart of participation in the Swedish Twin registry, birth years 1926-1958. PRS: polygenic risk scores; GWAS: genome-wide association studies; GP: general practitioner; LDSC: linkage-disequilibrium score regression.

\section{Affective traits}

Major depression is a derived variable based on a number of questions based on the Composite International Diagnostic Interview Short Form (CIDI-SF). Positive for major depression is given if there are: symptoms of major depression (dysphoria and anhedonia) and a CIDI-SF sum score $\geqslant 4$ or positive for antidepressant use [29].

Self-report anxiety was a positive answer to the question: "Have you ever had a period lasting one month or longer when most of the time you felt worried and anxious?"

A short form of the Eysenck Personality Questionnaire was used to measure neuroticism [30]. Neuroticism was used both as a continuous trait and as a categorical trait divided into approximate quartiles (scores: 0 $1,2,3-4,>5)$.

\section{Polygenic risk scores}

Single nucleotide polymorphism (SNP)-based genotyping was carried out using DNA from whole blood. Summary statistics from large GWAS (discovery sample) were used to compute genetic propensity scores 
for each trait in our own study population (target sample) using the individual genotypes. We calculated polygenic risk scores (PRS) for major depressive disorder (PRSMDD), primary anxiety disorders (PRSANX) and neuroticism $(\mathrm{PRSN})$ by summing risk alleles $(0,1$ or 2 alleles) at each SNP across the genome, weighted by individual SNP effect size derived from the discovery GWAS [18, 31, 32]. 8 sets of PRSs were computed based on the p-values of independent SNP associations in the discovery GWASs with the cut-off points ranging from GWAS significant signals starting at a p-value threshold ( $\mathrm{pT}$ ) of $5 \times 10^{-8}$ to incorporating all genetic signals, $\mathrm{p} \mathrm{T}=1$. This approach helps to reach the best balance between capturing meaningful genetic signals while reducing noise in our target sample. PRSs were then transformed to z-scores for use in further analyses (more details on PRS methods can be found in the supplementary material).

\section{Covariates}

Age at interview, sex, years of education, body mass index (BMI) and current smoking were derived from the 1998-2002 questionnaire.

\section{Statistical analysis \\ Questionnaire-based associations}

Multiple logistic regression models were used to determine the associations between each of the affective traits and asthma in the 23693 twins with data available on all traits of interest. Models included: 1) unadjusted; 2) adjusted for potential confounders of age, sex and education; 3) adjusted for age, sex, education and lifestyle mediators; 4) adjusted for age, sex, education and neuroticism; and 5) adjusted for age, sex, education and mutually adjusted for the other affective traits to see if any one trait was driving the observed associations. We also stratified the analysis by sex. Relatedness of twin pairs was accounted for by including a standard error sandwich estimator based on twin pair ID.

\section{PRS analysis}

First, the PRSs for affective traits in our sample were validated (more details can be found in the supplementary material). Then, the association between questionnaire-based asthma and the PRS for each affective trait was tested in separate logistic regression models, adjusting for age, sex, 10 genetic ancestry principle components, genotyping array and relatedness. Principle components are used to account for population stratification within the study population. The variance in asthma explained by each PRS was determined by comparison of Nagelkerke $\mathrm{R}^{2}$ from full (PRS and covariates) and reduced (covariates only) models. False discovery rate (FDR) at $<0.05$ was applied to deal with multiple testing over eight $p$-value thresholds. Next, the PRS pT explaining the greatest asthma variance was divided into quartiles based on distribution of PRS pT. Odds ratios were computed comparing the first quartile (i.e. the lowest genetic risk for affective traits) to other three quartiles in logistic regression models with asthma as an outcome, adjusted as above.

\section{Genetic correlations}

In a separate analysis based on GWAS summary statistics from the UK Biobank, genetic correlations between asthma and affective traits were estimated using LDSC without needing individual-level genotype information, as is the case with PRS approach. Association test statistics are regressed on their linkage-disequilibrium (LD) scores, a measure of each SNPs relationship with other variants [33]. The analyses were conducted in the LD Hub environment [34]. The most recently published GWAS summary statistics for asthma were used [35], restricted to samples with European descent. GWAS summary statistics for the affective traits were based on UK Biobank data available from www.nealelab.is/ uk-biobank/. UK Biobank summary results for affective traits were chosen for their broad inclusion of variables of interest. These included: two composite questions regarding a doctor or psychiatrist visit for anxiety/depression/tension/nerves; self-reported depression; self-reported anxiety/panic attacks and neuroticism score. Because neuroticism score is constructed from items tapping into depression symptoms and worry/anxiety [36], we also analysed all 12 neuroticism items to test which items show the highest genetic correlations with asthma. See the supplementary table E1 for a more detailed list of variables and GWAS sample sizes.

\section{Sensitivity analyses}

Associations were tested for probable asthma to check that the associations did not differ from our derived asthma variable that included asthma diagnoses, in case chronic obstructive pulmonary disease cases had been misdiagnosed as asthma.

Data management was conducted using SAS 9.4. Statistical analysis was conducted using STATA/IC 15.1.

The study was approved the Regional Ethical Review Board in Stockholm, Sweden. 
Results

The population of twins used for the questionnaire-based association analyses had a mean \pm SD age of $56.0 \pm 7.92$ years at testing in 1998-2002. The prevalence of asthma in this population was $7.9 \%(\mathrm{n}=2137)$, and $40 \%$ of these reported both a diagnosis and symptoms (probable asthma), 33\% only a diagnosis and $27 \%$ only symptoms. The prevalence of major depression was $20.1 \%$ and anxiety $19.9 \%$. The descriptive characteristics and affective traits for those with and without asthma is shown in table 1.

The population of twins used for the asthma-PRS association tests had a mean \pm SD age of $54.8 \pm 7.37$ years at testing in 1998-2002. The descriptive characteristics and prevalences were similar to the population used for the questionnaire-based analyses (table 1). The prevalence of asthma in this population was $9.0 \%$, major depression $21.7 \%$ and anxiety $20.5 \%$.

Questionnaire-based associations between each of major depression, anxiety, neuroticism (both continuous and categorical) and asthma are reported in table 2. The associations remained significant after adjusting for potential confounders (Model 2) and mediators (Model 3). Associations were slightly stronger for men than for women (table 2). When the models for major depression and anxiety were also adjusted for neuroticism, the associations slightly reduced but the significance stayed the same (Model 4; table 2). Mutual adjustment for all three affective traits seemed to attenuate the association between anxiety and asthma (particularly in females) and only slightly lowered the associations for major depression and neuroticism (continuous) (Model 5; table 2).

TABLE 1 Descriptive features of the Swedish Twin Registry study populations

\begin{tabular}{|c|c|c|c|c|}
\hline & \multicolumn{2}{|c|}{$\begin{array}{l}\text { Phenotypic analysis } \\
\text { population }\end{array}$} & \multicolumn{2}{|c|}{$\begin{array}{l}\text { Genetic analysis (polygenic } \\
\text { risk scores) population }\end{array}$} \\
\hline & $\begin{array}{l}\text { Asthma } \\
n=2135\end{array}$ & $\begin{array}{l}\text { No asthma } \\
n=21414\end{array}$ & $\begin{array}{l}\text { Asthma } \\
n=1448\end{array}$ & $\begin{array}{l}\text { No asthma } \\
n=14454\end{array}$ \\
\hline \multicolumn{5}{|l|}{ Age } \\
\hline Mean \pm SD & $55.03 \pm 7.98$ & $56.11 \pm 7.94$ & $53.57 \pm 7.13$ & $54.93 \pm 7.34$ \\
\hline Range & $41-74$ & $41-74$ & $41-74$ & $41-74$ \\
\hline \multicolumn{5}{|l|}{ Sex n $(\%)$} \\
\hline Males & $913(42.76)$ & $10167(47.48)$ & $600(41.44)$ & $6976(48.26)$ \\
\hline Females & $1222(57.24)$ & $11247(52.52)$ & $848(58.56)$ & 7478 (51.74) \\
\hline \multicolumn{5}{|c|}{ Level of education $\mathrm{n}(\%)$} \\
\hline$>9 \mathrm{yrs}$ & $567(26.56)$ & 5357 (25.02) & $460(31.77)$ & 4274 (29.57) \\
\hline$\leqslant 9 \mathrm{yrs}$ & 1417 (66.37) & $14809(69.16)$ & $878(60.64)$ & $9360(64.76)$ \\
\hline Missing & $151(7.07)$ & $1248(5.83)$ & $110(7.60)$ & $820(5.67)$ \\
\hline \multicolumn{5}{|l|}{ Smoking n (\%) } \\
\hline Yes & $410(19.2)$ & 4395 (20.52) & $228(15.75)$ & $2582(17.86)$ \\
\hline No & 1719 (80.52) & $16951(79.16)$ & 1220 (84.25) & 11862 (82.07) \\
\hline Missing & $6(0.28)$ & $68(0.32)$ & 0 & 10 (0.07) \\
\hline Body mass index & 2103 & 21041 & 1437 & 14335 \\
\hline Mean \pm SD & $25.81 \pm 4.08$ & $24.94 \pm 3.46$ & $25.55 \pm 3.72$ & $24.87 \pm 3.33$ \\
\hline Range & $14.7-47.8$ & $14.0-47.8$ & $15.0-45.5$ & $16.2-47.3$ \\
\hline \multicolumn{5}{|c|}{ Major depression n (\%) } \\
\hline Yes & $629(29.46)$ & $4103(19.16)$ & 450 (31.08) & 2999 (20.75) \\
\hline No & 1429 (66.93) & 16537 (77.23) & $980(67.68)$ & $11292(78.12)$ \\
\hline Missing & $77(3.61)$ & $774(3.61)$ & $18(1.24)$ & $163(1.13)$ \\
\hline \multicolumn{5}{|l|}{ Anxiety n (\%) } \\
\hline Yes & 562 (26.32) & $4103(19.16)$ & $402(27.76)$ & $2865(19.82)$ \\
\hline No & 1541 (72.18) & 17103 (79.87) & 1037 (71.62) & $11486(79.47)$ \\
\hline Missing & $32(1.50)$ & $208(0.97)$ & $9(0.62)$ & $103(0.71)$ \\
\hline \multicolumn{5}{|l|}{ Neuroticism } \\
\hline Subjects $n$ & 1902 & 19181 & 811 & 8463 \\
\hline Mean $\pm S D$ & $3.26 \pm 2.49$ & $2.73 \pm 2.30$ & $3.20 \pm 2.43$ & $2.70 \pm 2.27$ \\
\hline Range & $0-9$ & $0-9$ & $0-9$ & $0-9$ \\
\hline Quartile 1 & 575 (26.93) & 7177 (33.52) & $241(16.64)$ & 3192 (22.08) \\
\hline Quartile 2 & 294 (13.77) & $3136(14.64)$ & 136 (9.39) & $1373(9.50)$ \\
\hline Quartile 3 & 458 (21.45) & 4714 (22.01) & 201 (13.88) & 2108 (14.58) \\
\hline Quartile 4 & 575 (26.93) & 4154 (19.40) & $233(16.09)$ & 1790 (12.38) \\
\hline Missing & $233(10.91)$ & 2233 (10.43) & 637 (43.99) & $5991(41.45)$ \\
\hline
\end{tabular}


TABLE 2 Questionnaire-based associations between affective traits and asthma for the whole population and separately for men and women

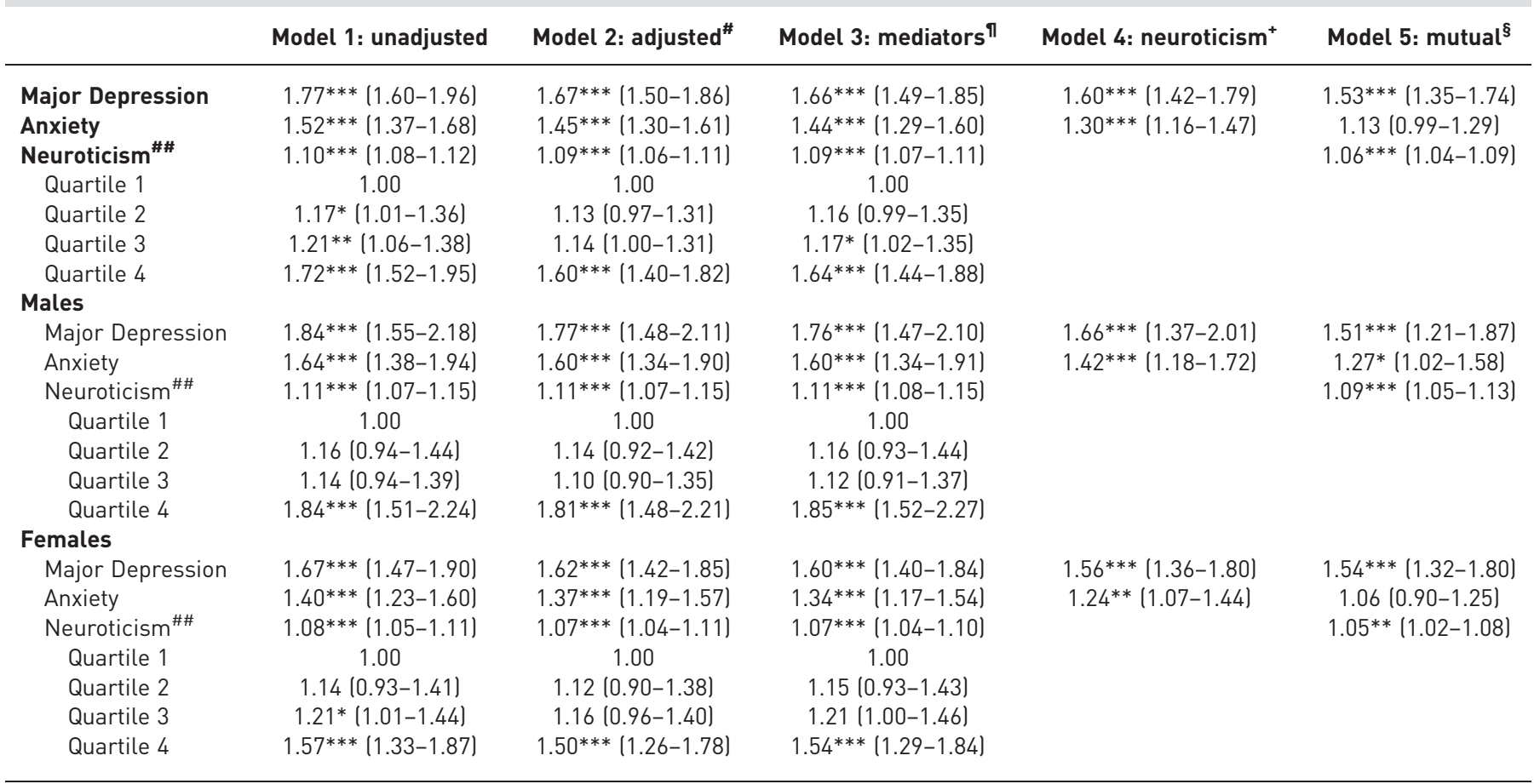

Data are presented as odds ratios with $95 \%$ confidence intervals. "\#: adjusted for age, sex, education level; ${ }^{\uparrow}$ : adjusted for age, sex, education level+body mass index and current smoking; ${ }^{+}$: adjusted for age, sex, education level+neuroticism; ${ }^{\S}$ : adjusted for age, sex, education level+the other affective traits; ${ }^{\# \#}$ : measured as a continuous trait. ${ }^{*}: \mathrm{p}<0.05 ;{ }^{* *}: \mathrm{p}<0.01 ;{ }^{* * *}: \mathrm{p}<0.001$.

The sensitivity analysis using the probable asthma definition did not change the effect sizes (supplementary table E2).

Validation of polygenic risk scores for major depression, anxiety and neuroticism confirmed them as significant predictors of each respective trait in our study population (figure E1). The PRSN explained up to $0.2 \%$ of the variance in asthma $(\mathrm{p}<0.001)$, which was about half of the variance explained by the genetic score in neuroticism itself (figure 2). No associations were seen for PRSMDD and PRSANX with asthma. One p T of each three affective traits was selected for subsequent analysis in quartiles, based on the highest variance explained in asthma, which was $\mathrm{p} \mathrm{T}<0.5$ for all three traits.

Quartile-based analysis revealed a dose-response effect for neuroticism risk scores, that is, with each increasing quartile of neuroticism score the risk of asthma increased (table 3). For example, individuals with the highest neuroticism scores in the top quartile had a $37 \%$ higher odds $(\mathrm{p}<0.001)$ of having asthma compared with the group with the lowest genetic risk. No elevated asthma risk was observed with increasing genetic predisposition for MDD or anxiety.

The LDSC found that asthma was genetically correlated with the composite questions regarding a visit to a doctor or psychiatrist for anxiety, depression, tension or nerves (table 4). There was also a modest genetic correlation of asthma with self-reported depression $(\mathrm{rg}=0.17)$, but not with anxiety or neuroticism score. Item-level analysis for neuroticism revealed genetic correlation between asthma and items tapping into depressed mood (fed-up feelings, loneliness) rather than worry/anxiety.

\section{Discussion}

In this study we attempted to triangulate the role of genetic influences on the associations between asthma and affective traits by first investigating phenotypic associations and then applying two different genetic methods (PRS approach and LDSC) to investigate shared genetic relationship between asthma and affective traits. To the best of our knowledge, this is the first time a genetic link between asthma and depression, anxiety and neuroticism has been investigated using genome-wide data. The phenotypic results found associations between asthma and the affective traits: major depression, anxiety and neuroticism. The PRS analysis using genotypes from a large twin population found modest associations between asthma and 


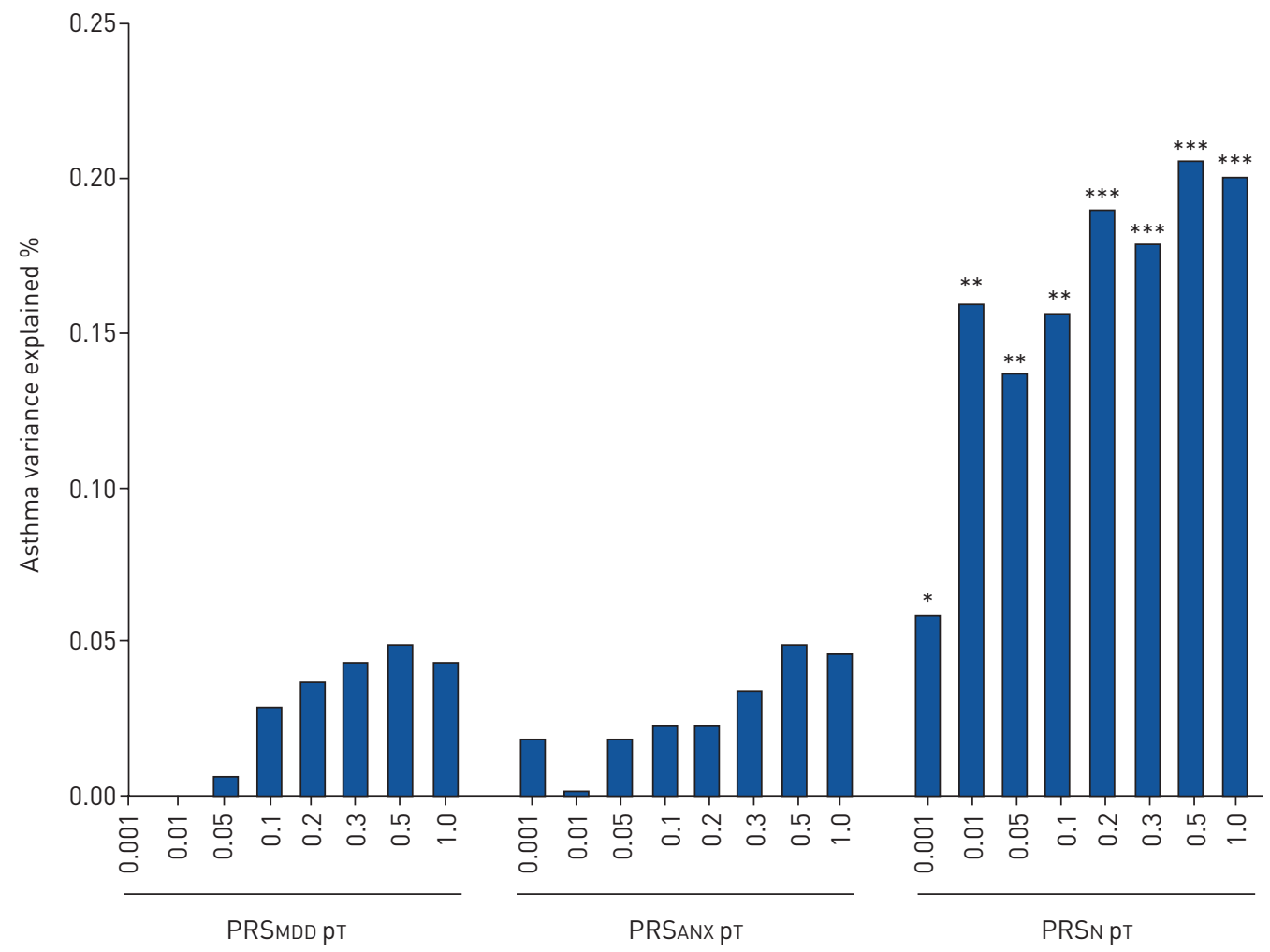

FIGURE 2 Polygenic risk scores (PRSs) for each of the affective traits explaining variance in asthma ( $n=15$ 908). MDD: major depressive disorder; ANX: primary anxiety disorders; $N$ : neuroticism; Uncorrected p-values: ${ }^{*}: p<0.05 ;{ }^{* *}: p<0.01 ;{ }^{* * *}: p<0.001$.

genetic susceptibility for neuroticism. The LDSC using GWAS summary statistics from UK Biobank detected genetic overlap between asthma and depression.

We found higher asthma risk in individuals with major depression, which is in line with previous research $[4,5,7]$. Modest but significant genetic correlation between self-reported depression and asthma was found using the LDSC approach, suggesting possible genetic overlap and shared biological pathways between these disorders. Possible biological candidates for depression and asthma comorbidity may include cytokine inflammatory factors such as interleukin (IL)-1, IL-4, IL-7 and tumour necrosis factor (TNF)- $\alpha$ [37]. However, we did not detect a significant association between asthma and PRS for major depressive disorder. The discordant results of the two methods could be potentially explained by different

TABLE 3 Associations of affective traits' polygenic risk score (PRS) quartiles (compared to the lowest genetic risk quartile) for each of the affective traits and asthma

\begin{tabular}{|c|c|c|c|c|c|}
\hline & \multirow[t]{2}{*}{ PRS $^{\#}$ quartile } & \multirow[t]{2}{*}{ OR } & \multicolumn{2}{|c|}{$95 \% \mathrm{Cl}$} & \multirow[t]{2}{*}{ p-value } \\
\hline & & & Lower & Upper & \\
\hline \multirow[t]{3}{*}{ Major depression } & Quartile 2 & 0.94 & 0.80 & 1.10 & 0.45 \\
\hline & Quartile 3 & 1.04 & 0.88 & 1.21 & 0.67 \\
\hline & Quartile 4 & 1.12 & 0.96 & 1.31 & 0.17 \\
\hline \multirow[t]{3}{*}{ Anxiety } & Quartile 2 & 1.12 & 0.96 & 1.32 & 0.16 \\
\hline & Quartile 3 & 1.14 & 0.97 & 1.34 & 0.11 \\
\hline & Quartile 4 & 1.12 & 0.96 & 1.32 & 0.16 \\
\hline \multirow[t]{3}{*}{ Neuroticism } & Quartile 2 & 1.18 & 1.00 & 1.39 & 0.05 \\
\hline & Quartile 3 & 1.25 & 1.06 & 1.48 & 0.01 \\
\hline & Quartile 4 & 1.37 & 1.17 & 1.61 & $<0.001$ \\
\hline
\end{tabular}

\#: the threshold probabilities selected were $\mathrm{p} T=0.5$ for all three traits. 
TABLE 4 Linkage-disequilibrium regression results between affective traits from UK Biobank and asthma from the Multiancestry Consortia association study [35]

\begin{tabular}{lcc} 
Disorder/trait & Genetic correlation & p-value \\
\hline $\begin{array}{l}\text { Seen doctor (general practitioner) for nerves, anxiety, } \\
\text { tension or depression }\end{array}$ & $\mathbf{0 . 1 2 6 1}$ & $\mathbf{0 . 0 0 2 5}$ \\
Seen a psychiatrist for nerves, anxiety, tension or depression & & $\mathbf{0 . 0 0 3 7}$ \\
Self-reported depression & $\mathbf{0 . 1 4 4 3}$ & $\mathbf{0 . 0 0 5 8}$ \\
Self-reported anxiety/panic attacks & 0.0919 & 0.3863 \\
Neuroticism score & 0.0734 & 0.176 \\
Mood swings & 0.0566 & 0.224 \\
Miserableness & 0.1022 & 0.0623 \\
Irritability & 0.009 & 0.878 \\
Sensitivity/hurt feelings & 0.0668 & 0.2001 \\
Fed-up feelings & $\mathbf{0 . 1 2 1 6}$ & $\mathbf{0 . 0 1 3 3}$ \\
Nervous feelings & 0.0084 & 0.8874 \\
Worrier/anxious feelings & 0.0554 & 0.3441 \\
Tense/highly strung & 0.0667 & 0.2595 \\
Worry too long after embarrassment & -0.0458 & 0.3789 \\
Suffer from nerves & 0.029 & 0.6426 \\
Loneliness, isolation & $\mathbf{0 . 1 3 7 4}$ & $\mathbf{0 . 0 0 3 3}$ \\
Guilty feelings & 0.0996 & 0.0658 \\
\hline
\end{tabular}

diagnostic criteria used in the two depression GWASs (diagnostic interview in PRS summary statistics versus self-reported depression in UK Biobank) or differences in their power to detect genetic signals, UK Biobank having a larger sample size. The ability of PRSs to detect associations increases with increasing power of the discovery GWAS $[38,39]$, therefore future replications are warranted with GWASs conducted in larger samples.

Anxiety has repeatedly been associated with asthma at the phenotypic level $[3,40,41]$. Our phenotypic results confirmed these associations in models where the effect of each affective trait was explored separately. However, considering the substantial correlation among anxiety, depression and neuroticism, we also conducted mutual adjustment for all affective traits. These results indicated a significant drop in the effect size of anxiety particularly in women, but not in the other affective traits. This could potentially indicate that depression and neuroticism may have a stronger influence on asthma compared to anxiety, particularly in women. Similar patterns were also seen in both analyses using genetic data. First, PRSs for anxiety failed to predict asthma in our sample. These results could potentially be explained by the lower power of the anxiety PRS compared to the neuroticism PRS, due to the smaller sample size of the original GWAS for anxiety. However, the genetic correlation method, LDSC, did not suffer from these limitations since data were derived from the well-powered UK Biobank sample. Yet the results suggested the same pattern: significant genetic correlations were found between asthma and self-reported depression as well as depression-related neuroticism items, but no genetic correlation was found with self-reported anxiety nor anxiety-related neuroticism items. Nevertheless, these results should be replicated in the future, when new GWASs with larger sample sizes on anxiety disorders emerge.

With regards to neuroticism, other studies have found up to a 15-year longitudinal association between neuroticism and the development of asthma $[5,13,15]$; our study extends this period up to 29 years. Even after this long follow-up time, the association between neuroticism and asthma was still rather strong (OR $1.60,95 \%$ CI 1.40-1.82 for the highest quartile of neuroticism scores), with effects similar to concurrently measured major depression (lowest versus highest quartile). Neuroticism is known to be a relatively stable personality trait $[16,17]$ and therefore may also be associated with asthma throughout the life span. We were able to demonstrate that PRSs for neuroticism were associated with asthma, which may provide some explanation for such long-term associations. The effects of such genetic composite scores are small, but the predictive ability of PRSs will improve with increasing power of GWASs to detect very small genetic signals. However, in the LDSC analysis we did not find evidence for genetic overlap between neuroticism and asthma despite data from a larger resource compared with the GWAS used for creating the neuroticism PRS. The neuroticism score as measured in UK Biobank and used in this study for LDSC, is composed of 12 heterogeneous items, some capturing more depressed mood and others nervousness and anxiety. Recently, it was reported that the genetic signal in neuroticism may originate from two different genetic clusters: depressed mood ("mood swings", "loneliness", "fed-up feelings", "miserableness") and worry ("nervous feelings", "worrier/anxious feelings", "tense/highly strung", "suffer from nerves") [36, 42]. 
Therefore, we investigated the genetic correlations for each neuroticism item separately and indeed found depression-related items to be more strongly associated with asthma compared to worry/anxiety related items. This further suggests that the genetic association may be stronger between asthma and depression than with anxiety.

This study has some limitations. The weak associations of anxiety in comparison to the other two affective traits could be influenced by the single-item anxiety measure that was used in the questionnaire-based association testing. The assessments of major depression and neuroticism were based on composite questions and therefore might be more robust. However, the LDSC analysis, which did not suffer from this limitation, still suggested the same pattern. Another limitation is that we were not able to test the direction of the association between asthma and affective traits, despite the longitudinal association found between neuroticism and asthma.

\section{Conclusion and future directions}

In conclusion, the observed comorbidity between asthma and the affective traits is in part due to genetic influences on the affective traits, and a moderate shared genetic risk of importance for both asthma and depression and neuroticism but not anxiety. This is the first study to observe a genetic susceptibility between asthma and affective traits. Replication studies will be advantageous in the future using more powerful GWAS once they become available. Furthermore, this study provides endorsement for future basic science research into the specific shared biological pathways for inflammatory and psychiatric disorders.

Acknowledgements: We acknowledge The Swedish Twin Registry at the Karolinska Insitutet for access to data and the database managers who have worked on the registry over several decades. We also acknowledge all participants in the Swedish Twin Registry without whom this study would not be possible. We thank Robert Karlsson at the Karolinska Institutet for biostatistical advice.

Conflict of interest: None declared.

Support statement: This work was supported by the Swedish Research Council through the Swedish Initiative for research on Microdata in the Social And Medical sciences (SIMSAM) framework (grant number 340-2013-5867); the Swedish Heart Lung Foundation; the Swedish Asthma and Allergy Association's Research Foundation, Swedish Research Council for Health, Working life and Welfare (FORTE, grant numbers 2013-8689, 2013-2292 and 2015-00289) and grants provided by the Stockholm County Council (ALF project). The Swedish Twin Registry is managed by Karolinska Institutet and receives funding through the Swedish Research Council under the grant number 2017-00641. Funding information for this article has been deposited with the Crossref Funder Registry.

\section{References}

1 To T, Stanojevic S, Moores G, et al. Global asthma prevalence in adults: findings from the cross-sectional world health survey. BMC Public Health 2012; 12: 204.

2 Soriano JB, Abajobir AA, Abate KH, et al. Global, regional, and national deaths, prevalence, disability-adjusted life years, and years lived with disability for chronic obstructive pulmonary disease and asthma, 1990-2015: a systematic analysis for the Global Burden of Disease Study 2015. Lancet Respir Med 2017; 5: 691-706.

3 Katon W, Richardson L, Lozano P, et al. The relationship of asthma and anxiety disorders. Psychosom Med 2004; 66: 349-355.

4 Brunner WM, Schreiner PJ, Sood A, et al. Depression and risk of incident asthma in adults. The CARDIA study. Am J Respir Crit Care Med 2014; 189: 1044-1051.

5 Loerbroks A, Herr RM, Subramanian S, et al. The association of asthma and wheezing with major depressive episodes: an analysis of 245727 women and men from 57 countries. Int J Epidemiol 2012; 41: 1436-1444.

6 Choi S, Kim SH, Lee JS. Association between depression and asthma in Korean adults. Asthma Allergy Proc 2017; 38: e37-e46.

7 Tedner SG, Lundholm C, Olsson H, et al. Depression or anxiety in adult twins is associated with asthma diagnosis but not with offspring asthma. Clin Exp Allergy 2016; 46: 803-812.

8 Patel PO, Patel MR, Baptist AP. Depression and asthma outcomes in older adults: results from the National Health and Nutrition Examination Survey. J Allergy Clin Immunol Pract 2017; 5: 1691-1697.e1.

9 Strine TW, Mokdad AH, Balluz LS, et al. Impact of depression and anxiety on quality of life, health behaviors, and asthma control among adults in the United States with asthma, 2006. J Asthma 2008; 45: 123-133.

10 Cluley S, Cochrane GM. Psychological disorder in asthma is associated with poor control and poor adherence to inhaled steroids. Respir Med 2001; 95: 37-39.

11 Chida Y, Hamer M, Steptoe A. A bidirectional relationship between psychosocial factors and atopic disorders: a systematic review and meta-analysis. Psychosom Med 2008; 70: 102-116.

12 Di Marco F, Santus P, Centanni S. Anxiety and depression in asthma. Curr Opin Pulm Med 2011; 17: 39-44.

13 Huovinen E, Kaprio J, Koskenvuo M. Asthma in relation to personality traits, life satisfaction, and stress: a prospective study among 11000 adults. Allergy 2001; 56: 971-977.

14 Loerbroks A, Apfelbacher CJ, Thayer JF, et al. Neuroticism, extraversion, stressful life events and asthma: a cohort study of middle-aged adults. Allergy 2009; 64: 1444-1450.

15 Loerbroks A, Li J, Bosch JA, et al. Personality and risk of adult asthma in a prospective cohort study. J Psychosom Res 2015; 79: 13-17. 
16 Terracciano A, McCrae RR, Costa PT Jr. Intra-individual change in personality stability and age. J Res Pers 2010; 44: 31-37.

17 Harris MA, Brett CE, Johnson W, et al. Personality stability from age 14 to age 77 years. Psychol Aging 2016; 31: 862-874.

18 Wray NR, Ripke S, Mattheisen M, et al. Genome-wide association analyses identify 44 risk variants and refine the genetic architecture of major depression. Nat Genet 2018; 50: 668-681.

19 Lo MT, Hinds DA, Tung JY, et al. Genome-wide analyses for personality traits identify six genomic loci and show correlations with psychiatric disorders. Nat Genet 2017; 49: 152-156.

20 Boomsma DI, van Beijsterveldt CEM, Hudziak JJ. Genetic and environmental influences on anxious/depression during childhood: a study from the Netherlands Twin Register. Genes, Brain Behav 2005; 4: 466-481.

21 Vukasovic T, Bratko D. Heritability of personality: a meta-analysis of behavior genetic studies. Psychol Bull 2015; 141: 769-785.

22 Polderman TJC, Benyamin B, de Leeuw CA, et al. Meta-analysis of the heritability of human traits based on fifty years of twin studies. Nat Genet 2015; 47: 702-709.

23 Wamboldt MZ, Hewitt J, Schmitz S, et al. Familial assocation between allergic disorders and depression in adult Finnish twins. Am J Med Genetics 2000; 96: 146-153.

24 Brew BK, Lundholm C, Gong T, et al. The familial aggregation of atopic diseases and depression or anxiety in children. Clin Exp Allergy 2018; 48: 703-711.

25 Lichtenstein P, De Faire U, Floderus B, et al. The Swedish Twin Registry: a unique resource for clinical, epidemiological and genetic studies. J Intern Med 2002; 252: 184-205.

26 Lichtenstein P, Sullivan PF, Cnattingius S, et al. The Swedish Twin Registry in the Third Millenium: an update. Twin Res Hum Genet 2006; 9: 875-882.

27 Magnusson PK, Almqvist C, Rahman I, et al. The Swedish Twin Registry: establishment of a biobank and other recent developments. Twin Res Hum Genet 2013; 16: 317-329.

28 Hallberg J, Dominicus A, Eriksson UK, et al. Interaction between smoking and genetic factors in the development of chronic bronchitis. Am J Respir Crit Care Med 2008; 177: 486-490.

29 Kendler KS, Gatz M, Gardner CO, et al. A Swedish national twin study of lifetime major depression. Am J Psych 2006; 163: 109-114.

30 Floderus B. Psycho-social factors in relation to coronary heart disease and associated risk factors. Nordisk Hygienisk Tidskrift 1974; 6: 7-148.

31 Otowa T, Hek K, Lee M, et al. Meta-analysis of genome-wide association studies of anxiety disorders. Mol Psychiatry 2016; 21: 1391-1399.

32 Okbay A, Baselmans BML, De Neve J-E, et al. Genetic variants associated with subjective well-being, depressive symptoms, and neuroticism identified through genome-wide analyses. Nat Genet 2016; 48: 624-633.

33 Bulik-Sullivan B, Finucane HK, Anttila V, et al. An atlas of genetic correlations across human diseases and traits. Nat Genet 2015; 47: 1236-1241.

34 Zheng J, Erzurumluoglu AM, Elsworth BL, et al. LD Hub: a centralized database and web interface to perform LD score regression that maximizes the potential of summary level GWAS data for SNP heritability and genetic correlation analysis. Bioinformatics 2017; 33: 272-279.

35 Demenais F, Margaritte-Jeannin P, Barnes KC, et al. Multiancestry association study identifies new asthma risk loci that colocalize with immune-cell enhancer marks. Nat Genet 2018; 50: 42-53.

36 Nagel M, Watanabe K, Stringer S, et al. Item-level analyses reveal genetic heterogeneity in neuroticism. Nat Commun 2018; 9: 905.

37 Jiang M, Qin P, Yang X. Comorbidity between depression and asthma via immune-inflammatory pathways: a meta-analysis. J Affect Disord 2014; 166: 22-29.

38 Dudbridge F. Power and predictive accuracy of polygenic risk scores. PLoS Genet 2013; 9: e1003348.

39 Wray NR, Lee SH, Mehta D, et al. Research review: polygenic methods and their application to psychiatric traits. J Child Psychol Psychiatry 2014; 55: 1068-1087.

40 Lee YC, Lee CT, Lai YR, et al. Association of asthma and anxiety: a nationwide population-based study in Taiwan. J Affect Disord 2016; 189: 98-105.

41 Gada E, Khan DA, DeFina LF, et al. The relationship between asthma and self-reported anxiety in a predominantly healthy adult population. Ann Allergy Asthma Immunol 2014; 112: 329-332.

42 Nagel M, Jansen PR, Stringer S, et al. Meta-analysis of genome-wide association studies for neuroticism in 449,484 individuals identifies novel genetic loci and pathways. Nat Genet 2018; 50: 920-927. 